\title{
Un viaje por las emociones en procesos de investigación feminista
}

\author{
A journey through emotions in feminist research
}

\author{
Dau García Dauder \\ Universidad Rey Juan Carlos de Madrid \\ dau.dauder@urjc.es (ESPAÑA) \\ Marisa G. Ruiz Trejo \\ IEI-Universidad Autónoma de Chiapas \\ marisaruiztrejoiei@gmail.com (MÉXICO)
}

Recibido: 18.062019

Aceptado: 07.09.2020

\section{RESUMEN}

En este artículo enfatizamos el valor epistémico de las emociones en los procesos de investigación (en el saber qué y en el saber cómo). Nos centramos no sólo en cómo las emociones de quien investiga afectan el proceso de investigación sino cómo el propio proceso afecta -emocionalmente- a las investigadoras. En concreto, explicamos las diferentes razones por las cuales es importante reconocer el papel de las emociones en los procesos de investigación: metodológicas e instrumentales, éticas, analíticas, políticas y sanadoras. También exponemos los inconvenientes de enfatizar las emociones en la investigación. Partiendo del concepto de "reflexividad fuerte" de las epistemologías feministas, proponemos un viaje para reflexionar sobre las emociones y sus diferentes implicaciones en una investigación académica: el impacto emocional de la investigación en la investigadora (especialmente cuando se trabaja con población vulnerable), el trabajo emocional que implica la investigación y, en concreto, el trabajo de campo (y los dilemas éticos que puede implicar), las emociones como datos o evidencia y el conocimiento emocionalmente sentido. 


\title{
PALABRAS CLAVE
} nistas.

Emociones, investigación, epistemologías feministas, metodologías femi-

\begin{abstract}
In this paper, we emphasize the epistemic value of emotions in the research process (to know what and know how). We focus not only on how the researcher's emotions affect the research process but also on how the process itself affects - emotionally- the researchers. Specifically, we explain the different reasons why it is important to recognize the role of emotions in research processes: methodological and instrumental, ethical, analytical, political and "healers". We also expose the drawbacks of emphasizing emotions in research. Starting from the concept of "strong reflexivity" of feminist epistemologies, we propose a journey through emotions and their different implications in feminist research: the emotional impact of research on the researcher (especially when working with vulnerable population); the "emotional work" involved in the research and, specifically, in the fieldwork (and the ethical dilemmas that may involve); emotions as data/evidence and emotionally sensed knowledge.
\end{abstract}

\section{KEY WORDS}

Emotions, research process, feminist epistemologies, feminist methodologies.

\section{INTRODUCCIÓN}

\subsection{El valor epistémico de las emociones}

Este texto tiene como objetivo reconocer el valor epistémico de las emociones en los procesos de investigación, partiendo fundamentalmente del marco teórico de las epistemologías feministas ${ }^{1}$. Con ello, en primer lugar, pretendemos promover la discusión teórica y epistemológica sobre qué entendemos por objetividad en investigación, entendida aquí como "conocimientos situados", "parcialidad" e "intersubjetividad u objetividad dinámica" (Collins, 1986; Haraway, 1995; Keller, 1991). Siguiendo las epistemologías feministas, consideramos que una "reflexividad fuerte" implica una "objetividad fuerte" (Harding, 1996), en

${ }^{1}$ Siguiendo la distinción clásica de Sandra Harding (1996), este artículo se encuadra concretamente dentro de las llamadas "epistemologías del punto de vista feminista" y "las posmodernas" (no en el marco del empirismo feminista). También es deudor de las aportaciones de las epistemologías feministas negras y decoloniales (Collins, 1986; Espinosa, 2017). 
la que repensar las emociones en los procesos de investigación dota de rigor a cuestiones ético-políticas muchas veces invisibilizadas. Por otro lado, el texto es producto de las reflexiones colectivas generadas a partir de diversos talleres realizados por las autoras, sobre "el cuerpo y las emociones en procesos de investigación", dirigidos en su mayoría a personas que están realizando su tesis doctoral (Ruiz-Trejo y García-Dauder, 2018). Consideramos que el contenido del mismo puede ser, a su vez, útil para fomentar la reflexividad en otros procesos y seguir contribuyendo al conocimiento colectivo.

Con todo ello, pretendemos contribuir a la ruptura de los dualismos del pensamiento occidental (mente/cuerpo, razón/emoción, público/privado, etc.), donde el segundo término queda infravalorado y asociado a lo femenino. El positivismo lógico ha entendido la objetividad como distanciamiento emocional. Además, a los científicos se les ha socializado para suprimir emociones, motivo por el cual históricamente se ha expulsado a las mujeres -asociadas a lo emocionaldel mundo de la ciencia (Harding, 1996; Keller, 1991), más si cabe, si estaban racializadas o pertenecían a comunidades indígenas, percibidas como ajenas a la "razón científica" (Espinosa, 2017). Las emociones han sido colocadas en el lado opuesto del conocimiento científico, en lo irracional, o en aquello que debe ser eliminado para que no interfiera, contamine o produzca "sesgos" inapropiados. Desde este paradigma androcéntrico, no hay lugar para el reconocimiento de las emociones en el trabajo de quien investiga, se ignoran o infravaloran. A lo sumo, se nombran para proteger la integridad de los datos o como parte del "fuera de campo" (Ruiz-Trejo y García-Dauder, 2018): son nombradas en los pasillos, en las charlas con amigas, o en las tutorías informales con directoras de tesis, pero sin relevancia para la producción de conocimiento o para aparecer en publicaciones ${ }^{2}$.

Ello ha supuesto una gran resistencia a discutir sobre el impacto emocional de una investigación (tanto en participantes de las investigaciones como en investigadoras). Recientemente, desde perspectivas críticas y cualitativas se han elaborado guías éticas de investigación donde se regula la relación o el vínculo que se establece con las personas "objeto" de estudio. Se previene así el impacto emocional negativo (o el posible daño) de la investigación en los sujetos investigados, especialmente, el riesgo emocional para las participantes en investigaciones sobre temas sensibles. Ello implica estrategias como asegurar el anonimato de las participantes, hacer entrevistas en lugares seguros, que sepan de antemano cómo será utilizada la información, reducir el estrés que implica, etc. También se tiene en cuenta que formar parte de una investigación puede ser beneficioso: puede tener un efecto catártico, de toma de conciencia o, incluso, empoderador o de denuncia (Chirix, 2003; Blázquez, Bustos y Restrepo, 2008).

${ }^{2}$ A lo largo del artículo, vamos a utilizar el femenino genérico: por referirse a persona; porque la bibliografía que utilizamos parte de las epistemologías feministas cuyas autoras son, en su mayoría, mujeres; y como forma de compensar la invisibilización del sujeto de conocimiento científico "mujeres". Por otro lado, corremos el riesgo de que se vuelva a asociar emociones a mujeres, lo cual no es nuestra intención. 
No obstante, no se piensa en el impacto emocional de una investigación en las propias investigadoras. "Parecería que el miedo a hablar por las experiencias o emociones de otros, nos ha incapacitado para hablar sobre nosotras mismas y nuestras emociones" (Widdowfield, 2000: 205). La investigadora no es solo un instrumento para recoger datos, reacciona y, como persona, tiene respuestas emocionales al campo. Como señalan Hubbard, Backett-Milburn y Kemmer (2001), podemos intentar que nuestros sentimientos no contaminen los datos, pero ¿podemos asegurar que nuestros datos no afecten nuestros sentimientos? No sólo la subjetividad y las emociones de quien investiga afectan al proceso de investigación, sino que también la investigadora se ve afectada por dicho proceso.

Las emociones (un amplio rango de ellas) están presentes y son inherentes al proceso investigador, se reconozcan o no; pero además pueden influir considerablemente en él. Las emociones acompañan los procesos de investigación (desde los primeros momentos en que se elige un tema hasta que se abandona un proyecto) y el trabajo de campo (la ansiedad o la empatía en una entrevista, por ejemplo). Pero además las emociones (de investigadoras y participantes) pueden ser datos y pueden ser recursos interpretativos. No solo conocemos a través de la cognición o el intelecto, también a través de las emociones (Game, 1997).

No obstante, corremos el riesgo de que, al nombrar, visibilizar y subrayar el papel no reconocido de las emociones en los procesos de investigación, contribuyamos a su vez al dualismo razón-emoción (aunque la intención sea la contraria). Pero no nombrarlas, refuerza el paradigma androcéntrico del racionalismo. Nos movemos en esa paradoja. En todo caso, nuestro objetivo es disolver el dualismo y politizar la jerarquía asociada (el subtexto de género), no defender un "emocionalismo" y mucho menos de carácter psicológico e individual. Además, queremos enfatizar que las emociones no solo son centrales en la producción de conocimiento, también en la movilización política. Tal como explica Diana Gómez (2019: 79), "ninguna transformación es posible sin abordar un cambio subjetivo que incluya el qué y el cómo sentimos; el alcance, las dimensiones y el ritmo de la transformación se complejizan de acuerdo a las particularidades afectivas de cada sujeto individual y colectivo".

Siguiendo esta línea, el presente artículo se encuadra en el marco teórico de las epistemologías feministas, y no tanto en los debates dentro de las ciencias sociales del llamado "giro afectivo". Usamos el término "emociones" en un sentido amplio que no distingue entre emociones, sentimientos o afectos ${ }^{3}$. No obstante, sí defendemos el carácter social de las emociones, se generan en contextos intersubjetivos y en comunidades; y, como ha subrayado la teoría feminista, son políticas. Pero, además, las emociones son herramientas cognitivas fundamentales para identificar relaciones de poder en los procesos de investigación (atravesadas por el género, la clase social, la raza, la edad o la orientación sexual de quien

${ }^{3}$ Aunque somos conscientes de que existe abundante literatura psicológica y social que los distingue, nuestro objetivo no es entrar en dicho debate. 
investiga, de los participantes, de los miembros de un equipo de investigación, etc.).

En definitiva, con este trabajo pretendemos repensar la relación inseparable entre conocimiento y emoción: las emociones son conocimiento, están implicadas en el saber qué y en el saber cómo (Jaggar, 1989; Holland, 2007). Esta idea es deudora de las epistemologías feministas. Junto con el reconocimiento de la parcialidad y los conocimientos situados como "objetividad fuerte", estas perspectivas reconocen el valor de conocimiento de las emociones en los procesos de investigación como parte de la "reflexividad fuerte" (Haraway, 1995; Harding, 1991; Guber, 2014). Para ello, se refieren al "conocimiento emocionalmente sentido" ("emotionally sensed knowledge") o a "formas emocionales de conocer" («emotional way of knowing") (Game, 1997; Hubbard et al., 2001). No es un conocimiento de las emociones, sino un conocimiento sentido a través de o por las emociones.

Nos referimos a una epistemología de las emociones donde la emoción (la empatía, el compromiso, etc.) contribuye a la comprensión y al conocimiento. Hilary Rose (1987), por ejemplo, reivindica el valor epistémico de la conjunción mano-cerebro-corazón; otras autoras defienden subjetividades -o puntos de vista- intrusas, traidoras, fronterizas (Smith, 1974; Collins, 1986; Anzaldúa, 1987), desde donde emana un conocimiento más crítico con los valores hegemónicos y menos indiferente a campos de ignorancia. "El conocimiento no es algo objetivo y ajeno a nuestros propios cuerpos, experiencias o emociones; es algo creado desde nuestras emociones y no solo desde nuestra cognición o intelecto" (Game, en Hubbard et al., 2001: 126). En una línea similar, los feminismos decoloniales han criticado la colonización de las emociones y han recuperado formas de senti-pensar (Méndez, López, Marcos y Osorio, 2013), de saber-hacer (Rivera Cusicanqui, 2010), o de conocimiento a través de lo oral (Bastian y Berrío, 2015), etc. Para Diana Gómez, los procesos sentipensantes son aquellos en los que: "razón y emoción se equilibran teniendo la capacidad de sentir sin quedar 'atrapado' en las emociones; ganar en conciencia y benevolencia de lo que mujeres y hombres sienten, piensan y hacen; y pensar sin que la razón dominante nuble la capacidad de empatía con las y los otros. Para esto se requiere, entonces, disputar el habitus emocional hegemónico de la modernidad/colonialidad patriarcal" (2019: 89).

Por eso, nuestra propuesta con este trabajo es crear espacios de reflexividad fuerte, por ejemplo talleres ${ }^{4}$, donde se puedan pensar colectivamente las emociones a lo largo de los procesos de investigación (Ruiz-Trejo y García-Dauder, 2018), no solo de los sujetos investigados sino también de quien investiga. Las investigaciones suelen mostrar los resultados de una experiencia, pero no los procesos donde las emociones impactan en nuestra comprensión de la realidad. Para ello, es importante "conceptualizar sentires" (Calla, en Ruiz-Trejo, 2014),

${ }^{4}$ La elaboración teórica de este texto es producto de la revisión bibliográfica sobre el tema, pero también de los talleres epistémico-corporales que hemos realizado en los últimos años y de entrevistas a investigadoras feministas. Agradecemos a todas ellas su participación y conocimiento. 
pero atender también a sentimientos inexpresables o inexpresados, a los silencios, etc. A su vez, es preciso considerar los conflictos entre pensamientos y emociones; o tener en cuenta la fluidez, complejidad y mutabilidad de sentimientos (Game, 1997; Bondi, 2005). Pero, ¿qué importancia tiene reconocer el papel de las emociones en los procesos de investigación? A continuación, señalamos razones pragmáticas, éticas, epistémicas, políticas e, incluso, sanadoras.

\subsection{Por qué analizar las emociones en los procesos de investigación}

Discutir sobre emociones en los procesos de investigación tiene sus dificultades e inconvenientes. Como advierte Rebekah Widdowfield (2000), artículos académicos en los que se habla de las emociones de quien investiga corren el riesgo de ser minusvalorados como "emocionales", subjetivos y sesgados. Otro riesgo es la sobre-exposición de quien escribe, que puede ser criticada por utilizar la investigación como terapia personal. Por otro lado, está la dificultad de convertir sentimientos en palabras o de evaluar el impacto de las emociones en el proceso de investigación (por ejemplo, cuando son sutiles o inconscientes). Por último, está el peligro de la reflexividad: privilegiar la voz de las investigadoras sobre quien investiga, es decir, cómo poder expresar el yo y las emociones sin que sea a expensas de otros yoes y otras historias (Widdowfield, 2000). En ese sentido, Elke Emerald y Lorelei Carpenter (2015) han señalado los riesgos de que la investigadora se "muestre vulnerable": si bien puede añadir otra dimensión al análisis, también puede alejar el tema central, ser auto-indulgente o aburrir con información personal que se perciba como poco interesante o irrelevante. En definitiva, la vulnerabilidad emocional puede ser incómoda, tanto para quien escribe como para quien lee. Entonces, ¿qué ventajas tendría incluir las emociones como conocimiento en un proceso de investigación?

En las últimas décadas, se ha atendido a las emociones de los sujetos investigados por razones éticas: es importante si queremos interactuar de forma sensible con las participantes, cuidar la relación y no provocarles daño con el uso instrumental de la investigación. Fundamentalmente, la literatura se ha centrado en emociones derivadas del contacto directo con los sujetos en investigaciones cualitativas (y mucho menos en las cuantitativas). También se ha analizado el impacto emocional positivo que puede tener una investigación para quien participa en ella, con procesos de toma de conciencia y reflexividad, de sentirse escuchadas y reconocidas, de dar y recibir afecto, de empoderarse, etc. (Chirix, 2003).

Por otro lado, es importante conocer cómo pueden afectar las emociones de quien investiga a la investigación por motivos prácticos o metodológicos. En primer lugar, porque pueden afectar a que un determinado tema sea investigado (porque te "toca" o te apasiona) y a la forma en que es investigado. Las emociones también pueden permitir construir una relación de confianza con las participantes y acceso a información. Pero, igualmente, pueden paralizar un proceso: por ejemplo, emociones derivadas de dilemas éticos o del sentimiento de que 
el trabajo de investigación no "vale la pena", no marca ninguna diferencia, etc. La comunidad académica apenas ofrece espacios para hablar abiertamente y de forma segura sobre las experiencias emocionales en la investigación (compartir experiencias positivas y negativas, dificultades, estrategias, etc.). Ello no solo tendría un valor preventivo (de parálisis y abandono, por ejemplo), sino también de apoyo colectivo y de mayor reflexividad sobre los procesos (Widdowfield, 2000; Bondi, 2005).

Esto nos lleva a una tercera justificación para tratar las emociones en investigación: puede favorecer el desarrollo de investigaciones más sanadoras y seguras. "Investigar acerca del afecto es vivir el afecto" (Chirix, 2003: 35) y es indagar sobre el cuerpo, en donde se experimentan e inscriben los afectos (Gómez, 2019). Discutir colectivamente sobre las emociones en una investigación puede tener un rol catártico, de desahogo, sin la sensación de soledad que acompaña a muchas investigadoras. Esto puede prevenir, por ejemplo, somatizaciones derivadas del riesgo emocional de algunas investigaciones (sobre todo, las que trabajan con temas sensibles o en contextos de violencia). Para el colectivo de Mujeres de Kaqla, la investigación requiere una reflexión teórico grupal en su metodología, "pero, sobre todo, terapias grupales de sanación para cambiar valores y actitudes de opresión hacia valores y actitudes de liberación" (Chirix, 2003: 36). Además, el intercambio de experiencias y estrategias puede ayudar al afrontamiento de emociones negativas y servir de apoyo y aprendizaje a investigadoras nóveles (Widdowfield, 2000).

Por último, y más importante, existen razones epistémicas para incluir las emociones en los procesos de investigación: porque lejos de interferir o contaminar una investigación, las emociones son herramientas y fuentes de información (Bastian y Berrío, 2015). Si se atienden, pueden mejorar la calidad de la misma. Como han señalado Emerald y Carpenter (2015), valorar la vulnerabilidad y situar la emoción en la investigación puede enriquecer nuestra comprensión del mundo social, al tiempo que nos cuidamos como investigadoras y preparamos a jóvenes investigadoras a afrontar sus emociones en estos procesos. Pero las emociones son además políticas, auténticos motores de transformación social, instrumentos para combatir abusos, para fomentar derechos, para cambiar la estructura de opresiones, para desatar nudos y abrir candados, para posicionarnos y repensarnos críticamente (Chirix, 2003; Leyva, 2015). En ese sentido, Mari Luz Esteban (2011) habla de "compromiso corporal" en la investigación feminista.

A continuación, vamos a desarrollar las cuatro principales áreas en las que la literatura ha analizado el papel de las emociones en la investigación. Pero principalmente nos vamos a centrar en las emociones de quien investiga: cómo la investigación le afecta o afecta a sus emociones y cómo sus emociones afectan la investigación. 


\section{LAS EMOCIONES EN EL PROCESO INVESTIGADOR}

\subsection{El impacto emocional de una investigación}

Determinadas investigaciones pueden tener un importante impacto emocional. En la investigación cualitativa, conocer a las personas y escuchar sus relatos rompe con la idea de objetividad y distancia emocional. La subjetividad y la conexión entre investigadora y participantes son componentes básicos en la relación de investigación (procesos emocionales como la identificación, intimidad, empatía, entendimiento, reciprocidad, respeto o confianza). El impacto emocional puede ser más evidente con las metodologías feministas que utilizan la "reflexividad fuerte", es decir, la preocupación por las relaciones de investigación, el cuidado hacia los grupos que investigamos, la atención a la subjetividad, a las relaciones de poder, a no dañar, a ofrecer algo de vuelta, etc. Las investigadoras feministas "se han centrado tanto en los efectos de su investigación en las participantes que se han olvidado de su propia vulnerabilidad emocional" (Sampson, Bloor y Fincham, 2008: 929).

Según la literatura, el impacto emocional en una investigación es mayor en trabajos cualitativos y que abordan temas sensibles. También está atravesado por la variable de género, más bien por las normas de sentimiento y expresión de emociones en una investigación asociadas a la masculinidad y feminidad (que se cruzan con las normas asociadas al rol de investigador científico). Como han señalado algunos trabajos, los investigadores varones suelen hablar más de riesgos físicos ("bodies on the line" cuando hacen investigación), mientras las investigadoras escriben más sobre los emocionales (Sampson et al., 2008). Aunque nos vamos a centrar en el riesgo emocional derivado de la investigación con temas sensibles y población en situación de vulnerabilidad (el que más se ha trabajado desde la literatura), no podemos olvidar otros impactos derivados de la investigación con población privilegiada, con élites de poder (machistas, racistas, clasistas, homófobas, etc.), y los riesgos emocionales o de violencia que también pueden sufrir las investigadoras (acoso, agresiones sexuales, discursos que hieren, etc.).

Rebecca Campbell (2001) habla de "investigaciones emocionalmente implicadas" para referirse a aquellas investigaciones que dejan un fuerte impacto emocional en quien investiga (por ejemplo, investigar el abuso sexual infantil o con supervivientes de violación, etc.). Se trata de temas ante los cuales una investigadora no puede permanecer inmune y es inevitable la empatía y la sensibilidad hacia el dolor de las participantes. En esos casos, en los que recoger información te "rompe el corazón" y es inevitable la implicación emocional, es difícil mantener un equilibro entre objetividad y sensibilidad (Coles y Mudaly, 2010; Rager, 2005). De hecho, a veces puede surgir el dilema entre el rol de investigadora o el rol terapéutico (Sampson et al., 2008).

$\mathrm{Al}$ escuchar los relatos de las participantes una y otra vez, la investigadora se ve expuesta a información impactante y emocionalmente fuerte. Los efectos por la exposición a material traumático pueden ir desde la insensibilización hasta 
una cierta "victimización secundaria". Cuando una investigadora escucha repetidamente a supervivientes de violación puede recordar su propia vulnerabilidad ("podría haber sido una de ellas") o generar miedos a que ella o algún familiar puedan ser víctimas de violencia sexual también. A veces, la investigadora puede recordar o revivir un abuso, si ella misma lo ha sufrido (Campbell, 2001). Jan Coles y Neerosh Mudaly (2010) hablan de "estrés traumático secundario" para referirse a síntomas físicos y emocionales en las investigadoras como respuesta acumulativa a entrevistas cara a cara con víctimas o a posteriores escuchas de transcripciones de material traumático. En este tipo de investigaciones, hacer entrevistas de forma repetida en poco tiempo puede provocar agotamiento emocional, no solo por los datos sino por la reacción de la investigadora a los datos.

Con este tipo de investigaciones, también pueden surgir conflictos morales, sentir que se está perdiendo sensibilidad tras escuchar tantos relatos duros o, incluso, alegrarse por el material de investigación que proporcionan las participantes. Se puede sentir que se está haciendo un uso instrumental de las participantes, aunque se les "dé voz" o se las escuche. O pueden aparecer sentimientos de fraude por no ofrecerles nada útil a cambio (Sampson et al., 2008).

Especialmente cuando la información confronta, o tiene resonancias en la investigadora, puede tener impacto en su propia vida, por ejemplo, en sus relaciones de pareja, familiares, provocar ansiedad, culpa, etc. Sampson et al. (2008) hablan de "retos personales asociados": por ejemplo, cuando una investigación sobre violencia de género hace que la investigadora re-evalúe su propia relación de pareja. La información recogida en las investigaciones también puede estimular recuerdos perturbadores o remover recuerdos dolorosos en la investigadora que le lleven a revisar eventos pasados emocionales. A través de una investigación, la investigadora puede revivir cosas que preferiría olvidar, pero también puede tener el efecto positivo de sacarlo a la luz y poder trabajar sobre ello.

Igualmente, el impacto emocional de una investigación puede ser positivo (tanto para las participantes como para la propia investigadora). Como desarrollaremos más adelante, las emociones de la investigadora son herramientas y fuentes de información. Pero no solo eso, los intercambios emocionales entre investigadora-participantes o entre investigadora-tema pueden tener un fuerte poder de transformación personal. Algunos procesos de investigación, especialmente cuando "tocan" a quien investiga, pueden convertirse en auténticos procesos de transformación personal y social (Diana Gómez en Ruiz-Trejo, 2016; Chirix, 2003).

\subsection{Las emociones a lo largo del trabajo de campo}

Se podría narrar un viaje por toda la amplia gama de emociones (positivas y negativas) que se viven a lo largo de un proceso de investigación, englobado a su vez en un proceso de carrera académica (Blakely, 2007). Emociones hacia los sujetos participantes, hacia el tema de investigación, hacia los lugares donde se investiga, hacia los datos, durante el trabajo de campo, la escritura, etc. Desde el 
apasionamiento con el que se comienza una investigación hasta el análisis frío de quien se aburre y se cansa con un tema que llega a su fin. Liora Nutov y Orit Hazzan (2011) han señalado la influencia de las emociones en la elección del tema de investigación y en el paradigma de investigación.

Seleccionar un tema de investigación implica emociones diferentes: o bien porque "te toca" o te apasiona, o bien porque hay motivos instrumentales o intelectuales en ello. Estas emociones son importantes, pues la investigación puede durar años en la carrera de quien investiga y son el motor de su implicación. Las emociones también pueden ser el punto de partida de una investigación. Por ejemplo, la ira o el dolor con las injusticias pueden ser el motor de una investigación feminista, también la esperanza. Muchas investigaciones parten de la incomodidad (Ahmed, 2015): problematizamos en una investigación lo que está problematizado en nuestra vida cotidiana. La selección del paradigma también implica emociones y un mayor o menor acople con la subjetividad de quien investiga (si se siente más cómodo con el distanciamiento emocional positivista o con la implicación emocional de la investigación co-labor).

Las emociones también pueden mover el abandono de una investigación: la soledad del proceso, la frustración y desmoralización por no cambiar las cosas, la culpa por provocar estrés en las participantes y no devolver nada a cambio, el aburrimiento, etc. Las emociones negativas también pueden tener efectos positivos, como señales de alerta que evitan un bloqueo o parálisis. Cuando se termina el trabajo de campo, si ha existido un compromiso emocional con el tema o con las participantes, se puede echar de menos; o, al contrario, se puede querer desconectar y que las participantes te sigan contactando o hablando (Sampson et al., 2008). También se puede experimentar rabia o alegría en función de las reacciones esperadas o no ante la publicación de resultados.

Más allá de estos momentos puntuales, la mayor parte de la literatura se ha centrado en las emociones en el trabajo de campo y, en concreto, el "trabajo emocional" que implica la relación investigadora-participantes (en etnografías, entrevistas, etc.). Nos vamos a centrar por ello en los debates desarrollados en torno al uso de la empatía en la investigación desde un punto de vista ético y feminista.

En un trabajo ya clásico, Ann Oakley (1981) se preguntaba si una feminista entrevistando a mujeres no era una "contradicción en sí misma". Esta autora defiende la tradición de las metodologías feministas y de procesos más igualitarios de investigación, de conocimiento intersubjetivo, donde se rompe la distinción jerárquica sujeto-objeto. En concreto, defiende el uso de la empatía, la intimidad y la reciprocidad en la entrevista feminista, apelando a la experiencia común entre mujeres ("sisterhood"). Es más, plantea que la entrevista puede llevar a una "transición hacia la amistad" ("transition to friendship"). No obstante, su propuesta ha sido matizada y criticada. Por un lado, se ha criticado el uso de la sororidad entre mujeres: las mujeres están atravesadas por otras variables que les puede hacer no empatizar (por edad, clase social, orientación sexual). Por otro lado, a veces el abuso de la empatía (Watson, 2009) puede cerrar el significado 
interpretativo de otras emociones: la no empatía muestra fragmentación, contradicción, de quien investiga, lo cual puede ser fuente de conocimiento.

En un artículo donde se preguntaba "¿puede existir una etnografía feminista?", Judith Stacey (1998) advierte que bajo la apariencia de un mayor respeto e igualdad con los sujetos de investigación se puede enmascarar una explotación más profunda y peligrosa. La empatía y promover la amistad también tienen el riesgo de la manipulación y la traición. Es más, plantea que cuanto mayor sea la intimidad o la aparente reciprocidad entre investigadora/participante, mayor será el daño o riesgo de explotación (mucho mayor que en los métodos positivistas "masculinos" según esta autora). El trabajo de campo implica una intrusión e intervención en un sistema de relaciones donde la investigadora es mucho más libre de abandonar, donde puede aparecer un conflicto de intereses (por ejemplo, información que se tiene pero que puede hacer daño), o la disonancia entre el trabajo de campo y el producto etnográfico (que, aunque se plantee como igualitario y recíproco, la autoría es solo de quien investiga). Una publicación es una intervención sobre la vida y las relaciones de los sujetos (que quedan expuestos a interpretaciones, juicios, etc.), negociar la narración final no quita el problema de la autoría, ni la vulnerabilidad en la que quedan los sujetos investigados (Cotterill, 1992; Stacey, 1998).

En "Amistad, vulnerabilidad y poder entrevistando a mujeres", Pamela Cotterill (1992) discute sobre la reciprocidad y el cuidado en las investigaciones feministas. Frente a la "transición hacia la amistad" de Oakley, propone la "extraña amigable" ("the friendly stranger"). A veces se habla más, precisamente, porque se percibe a quien entrevista como extraña. La oyente empática y comprensiva es también una extraña amigable que, a diferencia de la amiga, no ejerce control social. Para Cotteril (1992), Oakley no tiene en cuenta los dilemas morales implicados en la amistad en la entrevista: además del riesgo de manipulación, los juegos de vulnerabilidades (de las entrevistadas, pero también a veces de la entrevistadora), la culpa de la entrevistadora por escudriñar en la vida de la entrevistada u obtener información embarazosa o incómoda, etc. ¿Cómo reaccionar cuando la entrevistada se toma la entrevista como una conversación terapéutica al abrirse y contar temas dolorosos? ¿Cómo reaccionar cuando una participante no para de llorar o cuando, como reacción, a la entrevistadora se le caen las lágrimas? ¿Se muestran las emociones (se apaga la grabadora, se le toca con la mano, se le pregunta si prefiere interrumpir la entrevista) e incluso se cuenta información personal de vuelta? (a riesgo de que se viva con incomodidad por parte de la entrevistada). ¿O se reprimen, se cambia de tema y se evita la empatía para evitar situaciones emocionalmente intensas? La amistad con las participantes puede derivar en conflictos de rol entre amiga-investigadora, la sensación de "debería hacer algo por ella...", cómo gestionar información o la sensación de no estar recogiendo información de forma rigurosa (siendo más una conversación que una entrevista, etc.).

Abordando la "ética de la empatía", Janet Holland (2007) señala que una mujer feminista al mostrar empatía hacia las participantes puede conseguir confianza y explorar emociones y experiencias íntimas, pero también puede 
provocar que la entrevistada diga cosas que preferiría haber callado (lo cual la vulnerabiliza). "Quiero explorar el tema de la maternidad (...) pero me siento como una intrusa en la vida de Kim al incitarla de forma experta para que exponga sus capas vulnerables de pensamientos y sentimientos que había enterrado por tanto tiempo como forma de auto-protección" (Emerald y Carpenter, 2015: 3). Ello plantea el dilema feminista del uso de las emociones de forma instrumental (una "amistad fingida") o como una competencia académico-laboral más para extraer información. Lo cual no dejaría de ser otra forma de comercialización de las emociones. Holland (2007) se pregunta hasta qué punto se puede hablar de consentimiento informado en esos casos que se usan las emociones para sacar información. En todo caso, quizá el problema de fondo en todos estos debates sean las diferentes concepciones de qué se entiende por empatía y hasta qué punto debería de presuponer, por definición, el propio proceso de reflexividad sobre sus efectos.

Todo ello tiene que ver con la ética y con el trabajo o regulación emocional en investigación. ¿Cuándo mostrar emociones y cuándo no? ¿Y cómo hacerlo? Dickson-Swift, James, Kippen y Liamputtong (2009) describen las siguientes situaciones emocionales: cuando un relato de la participante hace recordar a quien investiga su propia experiencia y empatiza; cuando las participantes "se ponen" emocionales y no pueden evitar reaccionar, etc. Si un sujeto participante provoca la ira de la entrevistadora (por comentarios sexistas, racistas, homófobos, etc.) y suprime la expresión de la misma y calla, ¿está manipulando? (Holland, 2007). ¿O debería de expresarla? Para algunas, mostrarse abiertamente emocional (por ejemplo, llorar) es positivo porque la investigadora muestra que ha conectado; para otras es problemático, mejor llorar a solas ("fuera de campo") porque existen ciertas reglas de expresión en ciencia-academia que es preciso seguir: ser profesional implica no mostrar signos externos de emoción (enmascarar u ocultar una emoción frente a las participantes).

Liz Bondi (2005) alerta de los peligros de unir ética y emociones, de establecer "reglas de sentimientos o expresión de emociones" adecuados en investigación. Ello implica juicios normativos sobre lo que una investigadora debería de sentir (o no) en el proceso de investigación (por ejemplo, cuando se aconseja "que no se tomen tan personalmente una investigación"). Esta autora cuestiona la atribución de valores negativos o positivos a particulares estados emocionales, que no dejan de expresar expectativas normativas asociadas a regímenes emocionales y reglas de sentimientos en el ámbito académico.

Para Aranguren Romero (2008), sí es necesario una "ética de la escucha" cuando nos encontramos ante lo indecible y lo inenarrable, ante testimonios de experiencia y dolor límite. Se refiere a investigaciones sobre situaciones de violencia extrema política, donde rememorar experiencias de dolor o sufrimiento es enfrentarse a la fractura del lenguaje. Donde se da la paradoja de la necesidad de hablar, la urgencia de ser escuchado y la emergencia del silencio para preservar la intimidad o el dolor. Querer hablar para sanar y a la vez evitarlo. Ello implica reconocer el valor epistémico del silencio (Bastian y Berrío, 2015): lo que las personas saben o sienten, pero no dicen (por obviedad, por secreto, por miedo, 
porque no pueden). Y una escucha en resonancia, que se des-centra y re-sitúa, cuando la investigadora es invadida por el dolor de los demás.

En todo caso, la investigación y la relación con las participantes (los límites) implica una constante gestión del yo de quien investiga: por ejemplo, para establecer rapport o reciprocidad, para que se sienta bien o relajada la participante, para que la investigadora no proyecte sus emociones en la interpretación de los datos o para ser consciente de las mismas y utilizarlo como fuente y herramienta de investigación. "¿Dónde dibujo la línea entre la investigadora objetiva, la escuchadora empática y la investigadora como terapeuta?" (Emerald y Carpenter, 2015: 2). Las emociones, a su vez, son políticas también, no solo tienen valor ético o informativo.

Todo ello recoge los debates en torno a la experiencia de emociones en el trabajo de campo, unas emociones que son encarnadas y que van cambiando a lo largo del proceso de investigación. Gill Hubbard et al. (2001) describen varios ejemplos. Cuando recibir una información "te toca", te emociona, ¿cómo reaccionar? Cuando escuchar determinados discursos te hiere y te provoca rabia, ¿cómo reaccionar? Cuando sobre-empatizas, cuando te entra la tentación o la necesidad de intercambiar y compartir experiencias propias con participantes cual amiga o consejera, para apoyar, ¿cómo reaccionar? También puede aparecer estrés emocional, si se detesta a la gente que se investiga; ansiedad o impaciencia, si las participantes se "enrollan" o dan información no relevante; culpa y preocupación, si las investigadoras engañan o no cuentan del todo los objetivos de la investigación, o si quieren ayudar y se sienten fraude porque la investigación no es suficiente para un cambio en la vida de la gente. También pueden aparecer emociones positivas como la euforia después de hacer una entrevista porque te "da subidón".

Las emociones en el momento de devolución de información, o devoluciones recíprocas en procesos de investigación dialógica, son también un importante indicador evaluativo de los procesos de investigación y su horizontalidad (Bastian y Berrío, 2015).

Las emociones pueden ser muy variadas y cambiar a lo largo de todo el proceso, hacia las participantes o hacia el tema de investigación, durante el trabajo de campo, recogiendo los datos, en el proceso de escritura, etc. (Hubbard et al., 2001). A veces, como ya hemos señalado, pueden minar el sentido de autoeficacia como investigadora y como activista o, todo lo contrario, empoderar. En definitiva, posibilitar que una investigadora continúe o no investigando.

\subsection{Las emociones en el fuera de campo}

Libros como Domesticity in the making of modern science, Love and Capital o Who cooked Adam Smith's dinner, nos muestran el papel del espacio doméstico (el amor, los cuidados o la comida) como el fuera de campo constitutivo y necesario para el espacio de producción académica y científica. También las condiciones materiales de una investigadora, por ejemplo, la precariedad o la 
conciliación, son una importante trastienda en el ejercicio investigador. El espacio académico construye, por otro lado, sus propias reglas de sentimientos y expresión de emociones (relacionadas con la competitividad o la colaboración, la seguridad, la autoestima, etc.) que delimitan el marco de la investigación.

Margaret Rossiter (1992) ya nos describió a las pioneras científicas como una "contradicción en sus propios términos", en el sentido de que si eran científicas (y seguían las normas subjetivas asociadas a su rol) se las percibía como poco mujeres, y si eran mujeres (y seguían los mandatos de género) eran percibidas como poco científicas. Una situación que producía no pocos conflictos subjetivos de rol que obstaculizaban -y obstaculizan- la carrera académica de muchas mujeres. La vida emocional, asociada a lo irracional, lo femenino y el cuerpo, no tenía lugar en el proceso científico, quedando relegada al "fuera de campo", un trasfondo por otro lado constitutivo. Mucho menos los aspectos emocionales de la vida (extra-profesional) se podían colar en el trabajo y las carreras de las investigadoras. Todo ello, ha dificultado el análisis no sólo de cómo la investigación puede afectar (emocionalmente) la vida de quien investiga, sino también cómo la vida doméstica o personal de quien investiga puede afectar a la investigación. Pero datos y vidas están más relacionados de lo que nos permitimos reconocer, no hay más que ver la sección de agradecimientos en una tesis doctoral.

Centrándose en esta trastienda de la investigación, Nutov y Hazzan (2011) han analizado la forma en la que las doctorandas gestionan sus emociones durante su tesis doctoral ("feeling the doctorate"). Por ejemplo, se centran en las relaciones entre estudiante de doctorado y directora o director de tesis y la importancia de las emociones que genera dicha relación: cómo influyen en la elección mutua, pero también cómo las malas relaciones o la incompatibilidad (a veces de carácter o personalidad) constituye una de las principales causas de abandono de tesis. En ese sentido, la labor de tutorización y de apoyo emocional de quien dirige una tesis es valorada como igual de importante que su experiencia o conocimiento sobre el tema. Por otro lado, a veces se habla de procesos de "matar al padre" (o madre), utilizando la metáfora psicoanalítica, para representar cómo van cambiando las expectativas mutuas conforme avanza la carrera de quien hace el doctorado y se da un cambio de estatus: de estudiante a investigadora independiente (con su propia agenda, sus tiempos y tareas, etc.). Lo cual puede generar emociones encontradas en torno a relaciones de poder.

Nutov y Hazzan (2011) también se refieren a las emociones implicadas en las relaciones entre quien realiza un doctorado y su entorno de "iguales", emociones implicadas en una socialización académica en la que la cultura departamental puede fomentar la competitividad o la colaboración y el apoyo emocional entre pares. Por último, se refieren a las relaciones con el entorno de fuera de la academia y las emociones implicadas: por ejemplo, a veces una investigación puede quitar tiempo con la pareja, familia o amistades y no entenderse e, incluso, su contenido afectar a la vida personal de la investigadora. Pero también, al contrario, problemas personales pueden afectar el desarrollo de una investigación.

Por otro lado, revelar lo que normalmente es ocultado en una investigación, la "etnografía oculta", es cruzar fronteras emocionales en los relatos del trabajo 
de campo (Blackman, 2007). Este autor nos habla de comportamientos que permiten establecer confianza y rapport, pero que serían interpretados con recelo por la disciplina: por ejemplo, irse de fiesta, de borrachera o consumir drogas con participantes jóvenes. También se refiere a momentos de seducción o fantasías sexuales en el trabajo de campo. Hablar de ello puede prevenir situaciones de abuso de poder o acoso, establecer límites, etc. Obviamente, dicha etnografía oculta está atravesada por variables de género y poder. No es casual que investigadoras feministas hablen de cuidar, cocinar o bailar como parte de las actividades del trabajo de campo: "mezclando la deconstrucción con el baile" -"mixing deconstruction with dancing"- (Aparicio, 1998); "si no se puede bailar no es mi investigación" (Ruiz Trejo, 2015).

Por último, también aparecen emociones en los procesos de escritura o publicación (desde los bloqueos, hasta los conflictos en autorías colectivas). La subjetividad del neoliberalismo académico actual genera sus propios monstruos emocionales (derivados de la competitividad, el individualismo meritocrático, la burocracia, etc.).

\subsection{Las emociones como datos o recursos analíticos}

Las emociones de las participantes pueden ser utilizadas como datos o evidencia: si una persona llora mientras nos cuenta un suceso, ello nos aporta información sobre la importancia y el significado que le da al mismo. De igual modo, las emociones de la investigadora aportan información y conocimiento, son una parte necesaria del proceso reflexivo: otra "capa de datos". Como ya hemos desarrollado, las emociones durante el trabajo de campo, por ejemplo, en una entrevista, pueden aportar información útil sobre cómo actuar en la siguiente: si a la entrevistadora se le caen las lágrimas al escuchar la narración de un suceso doloroso, en la siguiente puede decidir contenerse y tomar distancia, lo que repercutirá a su vez en el tipo de información que sacará con su nueva actitud "menos empática" (Hubbard et al., 2001). También las experiencias emocionales en el trabajo de campo pueden aportar información sobre la identidad personal y profesional de la investigadora, por ejemplo, a partir de conflictos entre la identidad de género y la identidad científico-investigadora (cuidado versus instrumentalización).

Las emociones de la investigadora pueden constituir claves cognitivas o recursos interpretativos, para ello es preciso se coloque en un ejercicio reflexivo como "objeto" de investigación también. Por ejemplo, se pueden utilizar las emociones para hacer conscientes y examinar nuestras propias asunciones de partida; pueden actuar como espejos que visibilizan la posición de privilegio o vulnerabilidad de quien investiga. Las emociones también pueden actuar como amplificadoras, atrayendo nuestra atención más intensamente hacia algo que observamos (Emerald y Carpenter, 2015). Puede ser útil en términos analíticos identificar y reconocer procesos emocionales inconscientes en una relación de entrevista o en la interpretación de los datos: negaciones, proyecciones, identifi- 
caciones, etc. Identificar sentimientos a partir de omisiones o silencios. El análisis colectivo por parte de todo el equipo de estos elementos subjetivos puede evitar que se interpreten las palabras de las participantes a través de la respuesta emocional de una investigadora (Cylwik, 2001; Holland, 2007).

Las emociones pueden reflejar tensiones contextuales o diferencias en las subjetividades de la investigadora/participante (a diferencia de la empatía), lo cual puede ser usado como dato. Son especialmente importantes en situaciones de controversias: qué implica emocionalmente una "posición neutral" o posicionarse (Bondi, 2005). Emociones opuestas o contradictorias son una fuente de conocimiento privilegiada (Jaggar, 1989). Sara Ahmed (2015), por ejemplo, ha enfatizado el valor epistémico de la incomodidad o la desorientación en la investigación feminista. En un sentido similar, Virgina Vargas (2015) nos habla de la producción de conocimiento desde los nudos (emocionales), "deshilachando los entuertos". Las emociones son una importante herramienta cognitiva para identificar dinámicas de poder (Bastian y Berrío). En definitiva, nuestras emociones también son evidencia, nos pueden alertar sobre dónde poner la atención reflexiva, dónde poner cautela para que nuestro análisis no esté nublado por las emociones y dónde dejarse llevar, usando las emociones como amplificador, llevándonos a profundizar más (Emerald y Carpenter, 2015).

Larrea Killinger (2011: 11) se refiere a la "intensidad etnográfica" como "la actitud metodológica donde la reflexividad y la emoción ganan mayor fuerza durante el trabajo de campo, facilitando un mejor proceso de comprensión de los fenómenos observados". Esta autora se refiere a los "imponderables" del trabajo de campo como acontecimientos imprevisibles donde se refuerza la relación de confianza investigadora-participante. Se trata de nuevos espacios emotivos, encuentros de conocimiento mutuo, intercambio de experiencias y reflexividad compartida, donde se producen desplazamientos y las participantes se convierten en investigadoras. La intensidad etnográfica fortifica así el entendimiento a partir del estrechamiento de lazos y los vínculos afectivos: "me sentí entendida más que analizada" (en Chirix, 2003), entiende el campo como un espacio de interacción social, no como un lugar de recogida de datos. Audre Lorde (2003) habla de los "usos de lo erótico" (en bailes, encuentros, comidas), la implicación emocional o apasionada, como fuente de poder y conocimiento. Igualmente, los malos entendidos o problemas de comunicación durante el trabajo de campo, que evidencian la distancia entre investigadora y participantes, pueden aportar información sobre relaciones de poder, conflictos de rol (por ejemplo, entre investigadora y "amiga"), etc.

Es necesario una mayor formación y práctica para reconocer e interpretar las emociones como datos y no perder información. Igualmente, se necesitan compartir más experiencias prácticas de equipos de investigación. 


\section{CONCLUSIONES}

Las emociones (un amplio rango de ellas) son una parte integrante en todo el proceso de investigación. Las emociones en las participantes han sido consideradas como fuente de datos. Además, desde un punto de vista ético, se ha pretendido prevenir el riesgo emocional de los sujetos que participan en una investigación. No obstante, poco se ha trabajado sobre las emociones de las investigadoras, se espera se moderen o no se expresen para no interferir en los resultados. La escasa literatura al respecto se centra en las emociones de las investigadoras en investigaciones cualitativas y, especialmente, sobre temas sensibles, pero no cuantitativas. Bondi (2005) critica la idea de que el contacto cara a cara de la investigación cualitativa genera sentimientos más intensos que los números. La implicación y la demanda emocional puede ser la misma, pero de diferente manera: no hay más que pensar en la frustración, la alegría o el "fetichismo" que generan los programas estadísticos, los gráficos o tablas. Además, se puede pensar que el distanciamiento, la "frialdad" o la contención del positivismo son en sí mismos emocionales: "una pasión irracional hacia la racionalidad desapasionada" (Bondi, 2005: 234). Por otro lado, según esta autora, gran parte de la literatura se centra en emociones negativas 5 , en cómo se "gestionan" desde un punto de vista ético o instrumental, pero apenas sobre emociones positivas o sobre su valor epistémico y político en ejercicios de reflexividad (Bondi, 2005). Es necesario, por tanto, un mayor estudio sobre el papel de las emociones en procesos de investigación cuantitativa, así como sobre emociones no necesariamente "negativas".

En general, existe consenso en que el ámbito académico ha desatendido el valor epistémico de las emociones y, con ello también, el impacto emocional de la investigación sobre las investigadoras. En la cultura académica, en general, las emociones resultan incómodas, lo cual afecta a la joven investigadora con escasa formación para el nivel de implicación emocional que determinadas investigaciones demandan, e insegura para hablar abiertamente de sus experiencias con quien le dirige. Quien hace las entrevistas o el trabajo de campo no suele ser quien dirige los proyectos (y la investigadora menos experimentada puede ser la que lleve todo el peso emocional con los sujetos participantes), esa distancia hace que a veces no se consideren los riesgos emocionales (Hubbard et al., 2001). En definitiva, el riesgo emocional es mayor en investigadoras noveles que hacen investigaciones cualitativas sobre temas sensibles y conscientes del valor de la reflexividad.

Investigaciones realizadas desde organizaciones comunitarias o activistas han posibilitado espacios referentes de "senti-pensar" en el marco de procesos de transformación social: donde se reconoce el valor epistémico y político de las emociones en ejercicios de reflexividad colectiva (Méndez et al., 2013). Lejos de entender las emociones como un distractor social, la afectividad es una experien-

${ }^{5}$ Usamos la terminología de Bondi, pero entendemos que todas las emociones tienen una función y que habría que cuestionar también el adjetivo de "negativa o positiva". 
cia social significativa y una herramienta cognitiva. Por ello, se hacen visibles, para entender cómo funcionan dinámicas de poder, coloniales o de género (Chirix, 2003; Bastian y Berrío, 2015).

Por el contrario, en espacios académicos no existen espacios seguros (por ejemplo, talleres) donde se reconozca el papel de las emociones en la investigación, se analice el riesgo emocional y se validen las emociones como conocimiento. Es necesario que directoras de tesis, investigadoras principales o equipos de investigación adopten una responsabilidad colectiva en crear estrategias o recursos para trabajar de forma reflexiva, cuidadosa y segura con las emociones. Que no se queden en el espacio de las tutorías informales, el momento del café o conversaciones con amigas (Hubbard et al., 2001; Dickson-Swift et al., 2009).

En términos prácticos, algunas autoras proponen actividades como un viaje por las emociones a lo largo del proceso de investigación (Blakely, 2007) o, en una línea similar, un recorrido de recuerdos sobre prácticas de investigación y emociones asociadas (en relación con el tema o participantes, etc.) (Holland, 2014). En nuestros talleres, asociamos las emociones a itinerarios corporales (Esteban, 2008) en el proceso de investigación, o hacemos sesiones temáticas de atención reflexiva sobre alguna emoción en concreto (por ejemplo, un itinerario centrado en el dolor o en la ira o en la sorpresa, etc.).

Kathleen Rager (2016) propone una serie de recomendaciones prácticas para moderar el impacto emocional de una investigación sobre un tema tan sensible como el cáncer de mama. Si bien se trata de medidas individuales, de autocuidado, más que colectivas o institucionales. Una es el "diario": recoger pensamientos y sentimientos durante el trabajo de campo en un diario o en un blog como herramienta reflexiva o útil para afrontar reacciones. Otra medida son las reuniones con compañeras para el intercambio de experiencias y su discusión. A veces, también puede ayudar el consejo personal: hablar con otras personas, con la pareja, amigas o en terapia, etc. Para la autora, es de gran valor la evaluación o verificación de las participantes que asegura la calidad del estudio, pero además sirve para sentirse bien emocionalmente. "Mantener un equilibrio" es otra estrategia fundamental: la conciliación entre la vida personal y el trabajo de investigación (la vida es más que investigación), junto con el apoyo social y emocional de otras redes extra académicas. A estas estrategias de auto-cuidado, Hubbard et al. (2001) añaden: aproximarse a temas sensibles de forma gradual, no concentrar temporalmente las entrevistas cuando tienen una alta carga emocional, que haya más de una entrevistadora para distribuir la intensidad emocional y establecer un espacio para validar el trabajo emocional en la investigación.

A diferencia de otras profesiones (como el periodismo), poco se ha escrito sobre la gestión del riesgo de daño en investigadoras (Bloor, Finchman y Sampson, 2010). Los peligros del trabajo de campo no son solo ambientales (transmisión de enfermedades, aislamiento geográfico o social, por ejemplo), también situacionales (agresiones o amenazas cuando la presencia de investigadoras se percibe como incómoda y genera recelo, acoso sexual, etc.) y emocionales (trabajar temas dolorosos o traumáticos, o la resistencia o antagonismo de participantes, pueden tener consecuencias psicológicas y somatizaciones). Ser mujer 
incrementa el riesgo situacional y emocional (ya no digamos si se investigan temas "incómodos" como los feminicidios).

Pero es preciso no pensar estas medidas de protección de riesgo emocional o de trabajo reflexivo con las emociones como algo que se afronta en solitario. Son necesarias medidas de formación y protección institucionales: por ejemplo, tratar el tema en las agendas de formación de los programas de doctorado o en los proyectos, generar más bibliografía o guías prácticas. Como decíamos, crear dentro de los equipos de investigación -o en la comunidad de investigación más amplia- espacios seguros y de apoyo para hablar sobre emociones. En definitiva, crear una cultura de reflexividad colectiva donde las investigadoras puedan hablar sobre sus sentires en procesos de investigación, aprendan unas de otras sobre cómo manejar las emociones en diferentes contextos y utilicen las emociones como conocimiento para la transformación social.

\section{REFERENCIAS}

AHMED, Sarah (2015): La política cultural de las emociones, México, UNAM-PUEG. ANZALDÚA, Gloria (1987): La frontera/Borderlands: The new mestiza, San Francisco, Aunt Lute.

APARICIO, Frances (1998): Listening to salsa gender, Latin popular music, and Puerto Rican cultures, Hanover, University Press of New England.

ARANGUREN, Juan P. (2008): "El investigador ante lo indecible y lo inenarrable (una ética de la escucha)", Nómadas, 29, pp.20-33.

BASTIAN, Ángela Ixkic y BERRÍO, Lina R. (2015): "Saberes en diálogo: mujeres indígenas y académicas en la construcción del conocimiento", en Prácticas otras de conocimiento(s). Entre crisis, entre guerras. Tomo II, San Cristóbal de las Casas, Retos, pp.199-222.

BLACKMAN, Shane J. (2007): "Hidden ethnography: Crossing emotional borders in qualitative accounts of young people's lives", Sociology, 41(4), pp.699-716.

BLAKELY, Kristin (2007): "Reflections on the role of emotion in feminist research", International Journal of Qualitative Methods, 6(2), pp.59-68.

BLÁZQUEZ, Norma; BUSTOS, Olga; DELGADO, Gabriela y FERNÁNDEZ, Lourdes (2008): "Mujeres académicas: entre la ciencia y la vida", en Estudios Iberoamericanos de Género en Ciencia, Tecnología y Salud, Zaragoza, Prensas Universitarias de Zaragoza, pp.227-245.

BLOOR, Michael; FINCHAM, Ben; SAMPSON, Helen (2010): "Unprepared for the worst: Risks of harm for qualitative researchers", Methodological Innovations Online, 5(1), pp.45-55.

BONDI, Liz (2005): "The place of emotions in research: From partitioning emotion and reason to the emotional dynamics of research relationships", en Emotional geographies, Oxford, Ashgate, pp.231-242.

CAMPBELL, Rebecca (2001): Emotionally involved: The impact of researching rape, Nueva York, Routledge.

CHIRIX, Emma D. (ed.) (2003): Alas y raíces: afectividad de las mujeres mayas, Guatemala, Grupo de Mujeres Mayas Kaqla. 
COLLINS, Patricia H. (1986): "Learning from the outsider within: The sociological significance of Black feminist thought", Social Problems, 33(6), pp.s14-s32.

COLES, Jan; MUDALY, Neerosh (2010): "Staying safe: Strategies for qualitative child abuse researchers", Child Abuse Review, 19(1), pp.56-69.

COTTERILL, Pamela (1992): "Interviewing women: Issues of friendship, vulnerability, and power", Women's Studies International Forum, 15(5-6), pp.593-606.

CYLWIK, Helen (2001): "Notes from the field: emotions of place in the production and interpretation of text", International Journal of Social Research Methodology, 4(3), pp.243-250.

DICKSON-SWIFT, Virginia; JAMES, Erica. L.; KIPPEN, Sandra; LIAMPUTTONG, Pranee (2009): "Researching sensitive topics: Qualitative research as emotion work", Qualitative Research, 9(1), pp.61-79.

EMERALD, Elke; CARPENTER, Lorelei (2015): "Vulnerability and emotions in research: Risks, dilemmas and doubts", Qualitative Inquiry, 21(8), pp.741-750.

ESPINOSA, Yuderkys (2017): "Hacia la construcción de la historia de un (des)encuentro: la razón feminista y la agencia antirracista y decolonial en Abya Yala”, Praxis. Revista de Filosofía, 75, pp.1-14.

ESTEBAN, M. Luz (2008): "Etnografía, itinerarios corporales y cambio social: apuntes teóricos y metodológicos", en La materialidad de la identidad, Donostia, Hariadna Editorial, pp.135-158.

ESTEBAN, M. Luz (2011): Crítica al pensamiento amoroso, Barcelona, Bellaterra.

GAME, Ann (1997): "Sociology's emotions", Canadian Review of Sociology \& Anthropology, 34(4), pp.385-399.

GÓMEZ, Diana (2019): Emociones, epistemología y acción colectiva en contextos de violencia socio-política. Reflexiones breves de una experiencia de investigación feminista, en Otras formas de (des)aprender: investigación feminista en tiempos de violencia, resistencia y decolonialidad, Bilbao, UPV/EHU, pp.77-90.

GUBER, Rosana (comp.) (2014): Prácticas etnográficas. Ejercicios de reflexividad de antropólogas de campo, Buenos Aires, IDES/Miño y Dávila.

HARAWAY, Donna (1995): Ciencia, “cyborgs" y mujeres, Madrid, Cátedra.

HARDING, Sandra (1996): Ciencia y Feminismo, Madrid, Morata.

HOLLAND, Janet (2007): "Emotions and Research", International Journal of Social of Research Methodology, 10(3), pp.195-209.

HUBBARD, Gill; BACKETT-MILBURN, Kathryn; KEMMER, Debbie (2001): "Working with emotion: Issues for the researcher in fieldwork and teamwork", International Journal of Social Research Methodology, 4(2), pp.119-137.

JAGGAR, Alison M. (1989): "Love and knowledge: Emotion in feminist epistemology", Inquiry, 32(2), pp.151-176

KELLER, Evelyn F. (1991): Reflexiones sobre género y ciencia, Valencia, Edicions Alfons el Magnanim.

KILLINGER, Cristina L. (2011): “Intensidad etnográfica. Reflexividad y emoción en el trabajo de campo", Ankulegi. Revista de Antropología Social, 15, pp.11-22.

LEYVA, Xochitl (2015): “¿Academia versus activismo? Repensarnos desde y para la práctica teórica-política”, en Prácticas otras de conocimiento(s). Entre crisis, entre guerras. Tomo II, San Cristóbal de las Casas, Retos, pp.199-222.

LORDE, Audre (2003): La hermana, la extranjera, Madrid, Horas y Horas.

MÉNDEZ, Georgina; LÓPEZ, Juan; MARCOS, Sylvia; OSORIO, Carmen (coord..) (2013): Senti-pensar el género. Perspectivas desde los pueblos originarios, Guadalajara, La Casa del Mago. 
NUTOV, Liora; HAZZAN, Orit (2011): "Feeling the doctorate: Is doctoral research that studies the emotional labor of doctoral students possible", International Journal of Doctoral Studies, 6, pp.19-32.

OAKLEY, Ann (1981): "Interviewing women: A contradiction in terms", en Doing feminist research, Nueva York, Routledge, pp.30-61.

RAGER, Kathleen B. (2005): "Self-care and the qualitative researcher: When collecting data can break your heart", Educational Researcher, 34(4), pp.23-27.

RIVERA CUSICANQUI, Silvia (2010): Sociología de la imagen. Miradas ch’ixi desde la historia andina, Buenos Aires, Tinta Limón.

ROSE, Hilary (1987): "Hand, Brain and Heart", en Sex and Scientific Inquiry, Chicago, The University of Chicago Press, pp.265-282.

ROSSITER, Margaret (1992): Women scientists in America. Struggles and strategies to 1940, Baltimore, The Johns Hopkins University Press.

RUIZ TREJO, Marisa (2014): "Feminismos, memoria y justicia en América Latina. Entrevista a la antropóloga feminista Pamela Calla", Programa de Radio Contratiempo, número 210, disponible en http://www.contratiempohistoria.org/?p=4459

RUIZ TREJO, Marisa (2016): "Memoria de las emociones en la protesta social y en la construcción de paz en Colombia. Entrevista a la antropóloga feminista Diana Gómez”, Programa de Radio Contratiempo, número 278, disponible en http://www. contratiempohistoria.org/? $\mathrm{p}=6139$

RUIZ TREJO, Marisa (2015): Amor al aire. Antropología situada de las radios latinas en Madrid, Tesis doctoral, Universidad Autónoma de Madrid, España.

RUIZ TREJO, Marisa y GARCÍA-DAUDER, (S.) (2018): "Los talleres epistémico-corporales como herramientas reflexivas de la práctica etnográfica"”, Universitas $\mathrm{Hu}$ manística, 86, pp.55-82.

SAMPSON, Helen; BLOOR, Michael; FINCHMAN, Ben (2008): “A Price worth paying? Considering the 'cost' of reflexive research methods and the influence of feminist ways of doing", Sociology, 42(5), pp.919-933.

SMITH, Dorothy E. (1974): "Women's perspective as a radical critique of sociology", Sociological Inquiry, 44(1), pp.7-13.

STACEY, Judith (1998): “Can there a feminist etnography?”, Women’s Studies International Forum, 11(1), pp.21-27.

VARGAS, Virginia (2015): "Itinerario de los otros saberes", en Prácticas otras de conocimiento(s). Entre crisis, entre guerras. Tomo II, San Cristóbal de las Casas, Retos, pp.150-172.

WATSON, Cate (2009): “The 'impossible vanity': uses and abuses of empathy in qualitative inquiry", Qualitative Research, 9(1), pp.105-117.

WIDDOWFIELD, Rebekah (2000): "The place of emotions in academic research", Area, 32(2), pp.199-208. 
